\title{
Effects of Naltrexone Treatment for Alcohol-Related Disorders on Healthcare Costs in an Insured Population
}

\author{
Henry R. Kranzler, Leslie B. Montejano, Judith J. Stephenson, Shaohung Wang, \\ and David R. Gastfriend
}

\begin{abstract}
Objective: To determine the impact of treatment with oral naltrexone on healthcare costs in patients with alcohol-related disorders.

Methods: Using data from the MarketScan Commercial Claims and Encounters Database for 2000-2004, we identified a naltrexone group (with an alcohol-related diagnosis and at least one pharmacy claim for oral naltrexone) and two control groups. Alcohol controls had an alcoholrelated diagnosis and were not prescribed an alcoholism treatment medication. Nonalcohol controls had no alcohol-related diagnosis and no prescription for an alcoholism treatment medication. The control groups were matched three to one to the naltrexone group on demographic and other relevant measures. Healthcare expenditures were calculated for the 6-month periods before and after the index naltrexone drug claim (or matched date for controls). Univariate and multivariate analyses were used to compare the groups on key characteristics and on healthcare costs.

Results: Naltrexone patients ( $n=1,138 ; 62 \%$ men; mean age $45 \pm 11$ years) had significantly higher total healthcare expenditures in the pre-index period than either of the control groups. In the postindex period, naltrexone patients had a significantly smaller increase than alcohol controls in total alcohol-related expenditures. Total nonalcohol-related expenditures also increased significantly less for the naltrexone group than for the alcohol control group. Multivariate analyses showed that naltrexone treatment significantly reduced alcohol-related, nonalcohol-related, and total healthcare costs relative to alcohol controls.

Conclusions: Although prior to treatment patients with alcohol-related disorders had higher healthcare costs, treatment with oral naltrexone was associated with reductions both in alcoholrelated and nonalcohol-related healthcare costs.

Key Words: Naltrexone, Alcohol-Related Disorders, Healthcare Costs, Alcoholism, Pharmacoeconomics.
\end{abstract}

A LCOHOL USE DISORDERS (AUDs, which include alcohol abuse and alcohol dependence) are highly prevalent in the United States, with nearly $8.5 \%$ of the population meeting current (i.e., past-year) criteria for these disorders (Grant et al., 2004). AUDs are of considerable public health and economic significance, because of the high frequency of associated medical, psychiatric, family, legal, and work-related problems (Caetano and Cunradi, 1997). The annual cost of AUDs in the United States was estimated to be nearly $\$ 185$ billion for 1998 (Harwood, 2000), which in view of rising healthcare costs, is likely to be a substantial underestimate of current costs.

Department of Psychiatry (HRK), University of Connecticut School of Medicine, Farmington, Connecticut; Healthcare/Outcomes Research (LBM), Thomson Reuters, Ann Arbor, Michigan; HealthCore, Inc. (JJS, SW), Wilmington, Delaware; and Alkermes, Inc. (DRG), Cambridge, Massachusetts.

Received for publication June 29, 2009; accepted January 25, 2010.

Reprint requests: Henry $R$. Kranzler, Department of Psychiatry, University of Connecticut Health Center, Farmington, Connecticut 06030-2103; Tel.: 860-679-4151; Fax: 860-679-1316; E-mail: kranzler@psychiatry.uchc.edu

Copyright (c) 2010 by the Research Society on Alcoholism.

DOI: $10.1111 / j .1530-0277.2010 .01185 . x$
Despite the adverse effects of AUDs, the vast majority of individuals with these disorders never receive treatment (Cohen et al., 2007; Mojtabai et al., 2002; Rabinowitz et al., 1999; Regier et al., 1993; Wu et al., 2003). Recent efforts to improve both the efficacy and the acceptability of alcohol treatment have included the identification and testing of medications to treat alcohol dependence. This effort has resulted in the approval by the U.S. Food and Drug Administration of two oral medications (naltrexone and acamprosate), as well as a new, injectable, extended-release naltrexone formulation (Garbutt et al., 2005). These medications are in addition to disulfiram, which was approved during the mid-20th century.

A substantial literature shows that interventions to reduce heavy drinking or prevent relapse in alcoholism result in reduced healthcare costs, even when the cost of the treatment is accounted for (e.g., Holder, 1998; Holder and Blose, 1992; Parthasarathy et al., 2001; Pettinati et al., 1999). Pettinati and colleagues (1999) examined the effects of different modalities of alcohol treatment on this cost offset. Kranzler and colleagues (2008) used data from the 2000 to 2004 MarketScan $^{\circledR}$ Commercial Claims and Encounters Database to identify patients with alcohol-related claims who were prescribed oral naltrexone. Of 1,138 patients, $162(14.2 \%)$ were persistent in 
obtaining naltrexone, which was defined as having filled prescriptions for $\geq 80 \%$ of the 6-month treatment period. Nonpersistence with naltrexone was associated with a significantly greater use of costly healthcare services. That study did not, however, compare healthcare utilization by naltrexonetreated patients with that of other patients. Hence, this study compared healthcare costs for patients with an alcohol-related disorder who received naltrexone treatment with those of individuals with an alcohol-related disorder who were not prescribed pharmacotherapy and with a group of nonalcoholic individuals.

\section{METHODS}

The study used the MarketScan ${ }^{\circledR}$ Commercial Claims and Encounters (Commercial) Database for healthcare services incurred during the 5-year period January 1, 2000 through December 31, 2004. The healthcare business of the information company Thomson Reuters (New York, NY) constructs the MarketScan databases from the paid medical and prescription drug claims of individuals insured through its large self-insured employer and health plan clients located throughout the United States. In constructing the databases, information on healthcare claims from almost 100 payers, including self-insured employers, commercial insurance companies, Blue Cross and Blue Shield plans, and thirdparty administrators, is standardized and de-identified so that the resulting databases can be used to study real-world treatment patterns.

The resulting commercial database included the healthcare claims of 12 million insured individuals per year, on average, from 2000 through 2004. The claims come from all types of healthcare providers that submit information for payment to health plans and other payers, including hospitals, residential treatment facilities, physician offices, clinics, and retail and mail-order pharmacies. Each record in the database represents a unique healthcare service for which payment was requested. Individual database records (i.e., claims) were compiled at the patient level to study the healthcare utilization patterns of covered individuals. In addition to claims, basic demographic and health plan enrollment information is included in the database. Approximately $40 \%$ of health plans and other payers included in the database do not cover or they carve out substance abuse treatment. To avoid including patients with incomplete data, we limited the study sample to patients who had substance abuse coverage through their health plans.

\section{Sample Selection Criteria}

The naltrexone group had one or more claims for oral naltrexone between July 1, 2000 and June 30, 2004, the earliest of which occurring during this period was set as the study index date. Patients who were not continuously enrolled in a health plan for 6 months before and 6 months after the index date were excluded because their claims histories during the study period would be incomplete. Because the aim of the study was to compare healthcare utilization and expenditures before and after the initiation of oral naltrexone therapy, patients with claims for naltrexone (as well as disulfiram or acamprosate, which could confound the analyses) in the 3 months pre-index were excluded from the analysis. Patients with earlier pre-index claims for these drugs were retained in the sample because a 3-month gap in therapy was deemed to reflect a new course of treatment. Because the data were from the period preceding the availability of extended-release naltrexone for clinical use, there were no claims for that medication. To focus the analysis on patients who were receiving naltrexone to treat an alcohol use disorder, patients without one or more alcohol-related diagnoses in the 6 months before or after the index date were excluded.

Alcohol-related diagnoses were defined broadly using the criteria of the International Classification of Diseases, Ninth Revision, Clinical Modification (ICD-9-CM) and a list of diagnoses from Harwood and colleagues (1998). The following alcohol-related conditions were represented to ensure that all individuals with clinically significant effects of alcohol were included: Alcoholic psychoses; alcohol dependence syndrome; nondependent abuse of alcohol; alcoholic polyneuropathy; alcoholic cardiomyopathy; alcoholic gastritis; alcoholic fatty liver; acute alcoholic hepatitis; alcoholic cirrhosis of the liver; alcoholic liver damage, unspecified; fetal alcohol syndrome (in which case the mother was identified as the patient); excessive blood level of alcohol; toxic effects of ethyl alcohol; and accidental poisoning by alcohol.

Two control groups were selected for the analysis: (i) Alcohol controls were patients with one or more claims for an alcohol-related diagnosis; no drug claims for naltrexone, disulfiram or acamprosate; and 6 months of continuous enrollment prior to the index date and following the index date. (ii) Nonalcohol controls were patients with no claims for an alcohol-related diagnosis; no drug claims for naltrexone, disulfiram or acamprosate; and 6 months of continuous enrollment prior to the index date and following the index date. Because these

All patients in the database meeting the control sample criteria were eligible to be matched to the naltrexone sample for analysis. To ensure an even distribution of demographic characteristics that could influence healthcare utilization in all cohorts, thereby reducing confounding, both the alcohol controls and the nonalcohol controls were individually matched 3:1 to patients in the naltrexone group on the basis of gender, age, geographic region, relationship to primary insured, health plan type, and index quarter/year. Matches were assigned randomly from among all patients in the database who met the control sample criteria. The match ratio was chosen to enhance the capacity to detect potential cost differences because, although costs are generally highly skewed, even a small cost difference between groups could be meaningful. Because matching more than 3 controls to each case usually does not add power, a 3:1 match ratio was used (Mandrekar and Mandrekar, 2004).

\section{Variables}

Index Date. To determine the costs associated with naltrexone treatment, we chose an index date, which was the date from which we started to calculate healthcare costs and expenditures. For the naltrexone group, the index date was the date of the earliest naltrexone claim, between July 1, 2000 and June 30, 2004. The inclusion criteria required a 3-month "clean period" before the earliest naltrexone claim; this prevented confounding by the effects of recent pharmacotherapy for an alcohol-related diagnosis. Hence, the index date represented the initiation of naltrexone therapy, following at least 3 months with no evidence of alcohol-related pharmacotherapy (naltrexone, disulfiram, or acamprosate).

The index date for the control groups was randomly assigned such that the distribution of index dates by year and quarter did not differ significantly from that of the naltrexone cohort. The pre-index period was defined as the 6 months immediately preceding the index date. The postindex period started on the index date and continued for 6 months.

Demographic and Clinical Measures. The following standard demographic variables were available on insurance claims and included in the analysis: sex, age in years at index, geographic region (northeast, north central, south, west), relationship of patient to primary insured (self/employee, spouse, child/other), capitated insurance (yes, no), and insurance plan type (basic, comprehensive, EPO, HMO, noncapitated POS, PPO, capitated POS). These measures 
were selected because of their potential impact on healthcare expenditures.

To assess co-occurring medical conditions present in the pre-index and the postindex periods we used two claims-based comorbidity indices, the Charlson Comorbidity Index (CCI) (D'Hoore et al., 1996) and the Chronic Disease Score (CDS) (Von Korff et al., 1992). The CCI, one of the most widely used measures of the combined burden of disease or conditions (Valderas et al., 2009), is based on ICD9-CM diagnosis codes. The CDS is a risk-adjustment measure based on age, gender, and history of dispensed drugs that predicts hospitalization and can be used as an indicator of baseline comorbidity for control of confounding (Putnam et al., 2002). Although for both measures, higher scores indicate a greater burden of comorbidity, because they can yield slightly different results, both were used to control for the impact that the treatment of comorbid conditions has on total expenditures.

Alcohol-Related and Drug-Related Diagnoses. Patients were identified as having an alcohol-related condition if they had at least one medical claim with one of the alcohol-related ICD-9-CM diagnosis codes (Harwood et al., 1998). The diagnosis code could be present on any diagnosis field (primary or secondary) and on any type of claim [inpatient, emergency department (ED), outpatient]. Patients were identified as having a drug-related condition if they had at least one medical claim for one of the drug-related ICD-9-CM diagnosis codes (Harwood et al., 1998). Separate binary (yes/no) alcohol and drug diagnosis variables were created for the 6-month pre-index period and the 6-month study period.

Medical Care Costs. We calculated total healthcare payments using the gross payment field on inpatient, outpatient, and prescription claims. The gross payment represents the total amount eligible for payment for a service, including both the health plan and the patient (e.g., copayment, deductible) portions. All costs were adjusted to December 2004 dollars using the medical component of the Consumer Price Index (CPI). Separate figures were tallied for the 6-month pre-index period and the 6-month postindex period. In addition to providing a sum of total expenditures, we reported expenditures associated with specific subsets of claims. Subgroups examined included place or type of service (i.e., inpatient hospital, ED, outpatient, and outpatient pharmacy). We also divided expenditures into alcohol-related and nonalcohol-related, on the basis of the primary diagnosis code listed on each claim. Alcohol-related expenditures included inpatient, ED, and outpatient services claims where the primary diagnosis was an alcohol-related code, and drug claims for naltrexone. We considered only primary diagnoses in attributing costs as alcohol-related because of the potential to over count services if the secondary diagnosis referred to a mitigating factor rather than the reason for treatment. All other claims were considered nonalcohol related.

Medical Care Utilization. We examined medical care utilization rates by place or type of service (i.e., inpatient hospitalizations, ED visits, outpatient visits, and outpatient pharmacy prescriptions). For each place or type of service, we created a binary (yes/no) variable denoting the presence of any service and a continuous variable to record the number of relevant services. As with costs, utilization was examined overall and for alcohol-related services as identified by diagnoses on the claims.

In addition, we identified patients with certain types of alcoholrelated treatment, including individual psychotherapy, group psychotherapy, outpatient detoxification, and inpatient detoxification. This information was obtained for both the 6-month pre-index period and the 6-month postindex period. Procedure codes present on claims were used to identify patients with these services. In addition, MarketScan place of service codes were used to differentiate inpatient and outpatient detoxification.

\section{Descriptive Analyses}

Descriptive information included the demographic and clinical characteristics of the naltrexone group and both control groups, as well as their utilization and expenditures in the pre-index period and study period. Dependent variables also included utilization and cost measures, as well as specific measures of alcohol-related medical complications. Utilization included: (i) number of outpatient visits, (ii) number of hospitalizations, (iii) number of days hospitalized, (iv) number of ED visits, (v) number of prescriptions, and (vi) sum of days supplied across all prescriptions. Cost measures were calculated as paid claims after discounts. Cost measures included: (i) total medical care expenditures, (ii) outpatient expenditures, (iii) inpatient expenditures, (iv) ED expenditures, and (v) pharmaceutical expenditures.

Statistical testing was used to examine differences between the naltrexone and control samples. $t$-tests were used to analyze normally distributed continuous variables, Wilcoxon rank-sum tests to analyze non-normally distributed continuous variables, and chi-square tests were used to analyze categorical variables. To account for withinsubject correlation, paired t-tests were used to test the difference between the pre-index and postindex period expenditures.

\section{Multivariate Analyses}

Multivariate analyses were used to provide cost estimates that control for confounding factors. The final specification of the multivariate models was driven, in part, by the results of the descriptive analysis. Difference-in-difference (DID) regression analyses were used to generate a more accurate estimate of the total expenditure impact of naltrexone treatment.

Each individual had two observations: one in the period before the index date and one in the period after the index date. The generalized estimating equation (GEE) method was used to account for correlation between repeated measures across individuals. Nonlinear specification of the cost equation was estimated to account for the non-normal distribution of the error term (Liang and Zeger, 1986).

The general specification of the DID regression was as follows: Total $\$=\mathrm{f}$ (T, Alcohol Control, T*Alcohol Control, Nonalcohol Control, $\mathrm{T}^{*}$ Nonalcohol Control, $\left.\mathrm{X}\right)$.

In this specification, Total $\$$ refers to total healthcare expenditures in the pre-index and postindex periods (measured separately for each patient). The letter $\mathrm{f}$ refers to the assumption of a formal, empirical (i.e., exponential) relationship between healthcare expenditures and the factors in parentheses. " $\mathrm{T}$ " indicates whether the outcome is measured before or after treatment. "Alcohol Control" is a binary indicator denoting individuals with an alcohol diagnosis who did not receive treatment. The variable labeled "T*Alcohol Control" denotes the interaction between postindex expenditures and an alcohol diagnosis without treatment. "Nonalcohol Control" is a binary indicator denoting individuals without an alcohol diagnosis. The variable labeled "T*Nonalcohol Control" denotes the interaction between postindex expenditures and no alcohol diagnosis. $\mathrm{X}$ refers to all other covariates. In addition to the demographic and clinical characteristics included in Table 1, covariates included health plan type (indemnity, preferred provider organization, health maintenance organization); the primary insured's employee type (salary or hourly) and status (actively employed or retired); the median income in the 3-digit zip code area corresponding to the patient's residence; and binary indicators for a pre-index substance abuse diagnosis, pre-index psychotherapy, and postindex psychotherapy.

Ultimately, DID regression analyses, using the equation below, provided an estimate of the total impact on expenditures of naltrexone treatment.

$$
D I D=\left(\hat{C}_{\text {Naltrexone Post }}-\hat{C}_{\text {Naltrexone Pre }}\right)-\left(\hat{C}_{\text {Control Post }}-\hat{C}_{\text {Control Pre }}\right)
$$

This DID design compared the difference in healthcare expenditures between the 6-month pre-index period and the 6-month 
Table 1. Demographic and Clinical Characteristics of Naltrexone Patients and Controls (PostMatching)

\begin{tabular}{|c|c|c|c|c|c|}
\hline & $\begin{array}{l}\text { Naltrexone group } \\
\quad(N=1,138)\end{array}$ & $\begin{array}{l}\text { Alcohol controls } \\
(N=3,411)\end{array}$ & $p$-value ${ }^{a}$ & $\begin{array}{l}\text { Nonalcohol controls } \\
\qquad(N=3,410)\end{array}$ & $p$-value ${ }^{b}$ \\
\hline \multicolumn{6}{|l|}{ Gender (\%) } \\
\hline Male & $61.6 \%$ & $62 \%$ & 0.9965 & $62 \%$ & 0.9936 \\
\hline Female & $38.4 \%$ & $38 \%$ & 0.9955 & $38 \%$ & 0.9919 \\
\hline Mean age (SD) & $44.9(10.6)$ & $44.6(11.5)$ & 0.4000 & $44.8(11.0)$ & 0.6804 \\
\hline \multicolumn{6}{|l|}{ Geographic region (\%) } \\
\hline Northeast & $24.3 \%$ & $24.3 \%$ & 0.9982 & $24.2 \%$ & 0.9799 \\
\hline North Central & $36.4 \%$ & $36.4 \%$ & 0.9941 & $36.4 \%$ & 0.9921 \\
\hline South & $26.8 \%$ & $25.7 \%$ & 0.9921 & $25.8 \%$ & 0.9934 \\
\hline West & $13.6 \%$ & $13.6 \%$ & 0.9964 & $13.6 \%$ & 0.9952 \\
\hline \multicolumn{6}{|c|}{ Relationship to primary insured (\%) } \\
\hline Self (Insured Employee) & $60.2 \%$ & $64.4 \%$ & 0.4629 & $65.0 \%$ & 0.4016 \\
\hline Spouse & $33.7 \%$ & $26.6 \%$ & 0.0716 & $29.7 \%$ & 0.3247 \\
\hline Child/other & $6.2 \%$ & $9.1 \%$ & 0.1382 & $5.4 \%$ & 0.6488 \\
\hline \multicolumn{6}{|l|}{ Insurance plan type (\%) } \\
\hline Capitated & $27.8 \%$ & $30.7 \%$ & 0.4463 & $28.7 \%$ & 0.7991 \\
\hline Noncapitated & $71.9 \%$ & $69.2 \%$ & 0.6551 & $71.1 \%$ & 0.8919 \\
\hline Missing/unknown & $0.4 \%$ & $0.1 \%$ & 0.4335 & $0.2 \%$ & 0.6995 \\
\hline \multicolumn{6}{|l|}{ Index year (\%) } \\
\hline 2000 & $12.6 \%$ & $12.7 \%$ & 0.9965 & $12.7 \%$ & 0.9954 \\
\hline 2001 & $26.5 \%$ & $26.4 \%$ & 0.9987 & $26.4 \%$ & 0.9813 \\
\hline 2002 & $30.0 \%$ & $30.0 \%$ & 0.9946 & $30.0 \%$ & 0.9929 \\
\hline 2003 & $25.0 \%$ & $25.0 \%$ & 0.9951 & $25.0 \%$ & 0.9935 \\
\hline 2004 & $6.0 \%$ & $5.9 \%$ & 0.9757 & $6.0 \%$ & 0.9968 \\
\hline \multicolumn{6}{|c|}{ Charlson comorbidity index (CCl) mean (SD) } \\
\hline Pre-index period & $0.54(1.01)$ & $0.44(1.10)$ & 0.0026 & $0.21(0.72)$ & $<0.0001$ \\
\hline Postindex period & $0.58(1.20)$ & $0.76(1.47)$ & $<0.0001$ & $0.23(0.80)$ & $<0.0001$ \\
\hline \multicolumn{6}{|c|}{ Chronic disease score (CDS) mean (SD) } \\
\hline Pre-index period & $1.44(1.89)$ & $0.98(1.72)$ & $<0.0001$ & $0.58(1.33)$ & $<0.0001$ \\
\hline Postindex period & $0.91(1.67)$ & $0.97(1.74)$ & 0.2582 & $0.51(1.25)$ & $<0.0001$ \\
\hline
\end{tabular}

${ }^{\text {a}}$ Comparison of naltrexone group and alcohol controls.

${ }^{\mathrm{b}}$ Comparison of naltrexone group and nonalcohol controls.

study period for the naltrexone group to the same difference in the other patient groups. The DID analysis also allowed us to control for differences in mean baseline expenditures that could be attributed to the health conditions that we did not measure.

\section{RESULTS}

\section{Univariate Analyses}

There were 1,138 patients in the naltrexone group. Following matching, there were 3,411 alcohol controls and 3,410 nonalcohol controls. Because appropriate matches could not be found for a few naltrexone patients, both control groups were just short of a complete 3:1 match.

The demographic characteristics of the three groups are presented in Table 1. Sixty-two percent of the patients were men and the mean age was 45 years. The sample was distributed across the four geographic regions as follows: $24 \%$ resided in the northeast, $36 \%$ in the north central region, $26 \%$ in the south and $14 \%$ in the west. Sixty percent were employees, $34 \%$ spouses of insured employees, and $6 \%$ were children or other dependents, nearly all of whom were 18 years of age or older. Most $(72 \%)$ had noncapitated insurance coverage. Several different insurance plan types were represented in the sample, the most common of which was the preferred provider organization (PPO), with one-third of patients having this plan type. Some patients had index dates in each year from 2000 through 2004, but most of the sample had index dates in 2001, 2002, or 2003. As would be expected given the matching, the control groups did not differ significantly from the naltrexone group on any demographic variable.

As shown in Table 1, although all groups had low comorbidity scores during the pre-index period, the naltrexone group had more comorbid medical conditions than either control group, as evidenced by significantly higher CCI and CDS scores. Comorbidity scores were also generally low in all three samples during the postindex period. However, during this time, the CCI score of the naltrexone group was significantly lower than that of the alcohol controls, but significantly higher than that of the nonalcohol controls. Similar trends were evident for the CDS scores. More naltrexone patients than controls had drug-related claims in both the pre- and postindex periods (data not shown).

The corresponding expenditure results were similar, as shown in Fig. 1. Mean pre-index alcohol-related expenditures were significantly higher for the naltrexone group than the alcohol controls $(\$ 1,352$ vs. $\$ 68, p<0.001)$. (Expenditures related to naltrexone, disulfiram or acamprosate during this period amounted to only $\$ 13$ of total expenditures for the naltrexone group; data not shown.) Naltrexone patients also had higher mean pre-index nonalcohol-related ( $\$ 3,478$ vs. $\$ 2,435$, $p<0.001$ ) and total (alcohol-related plus nonalcohol-related) 


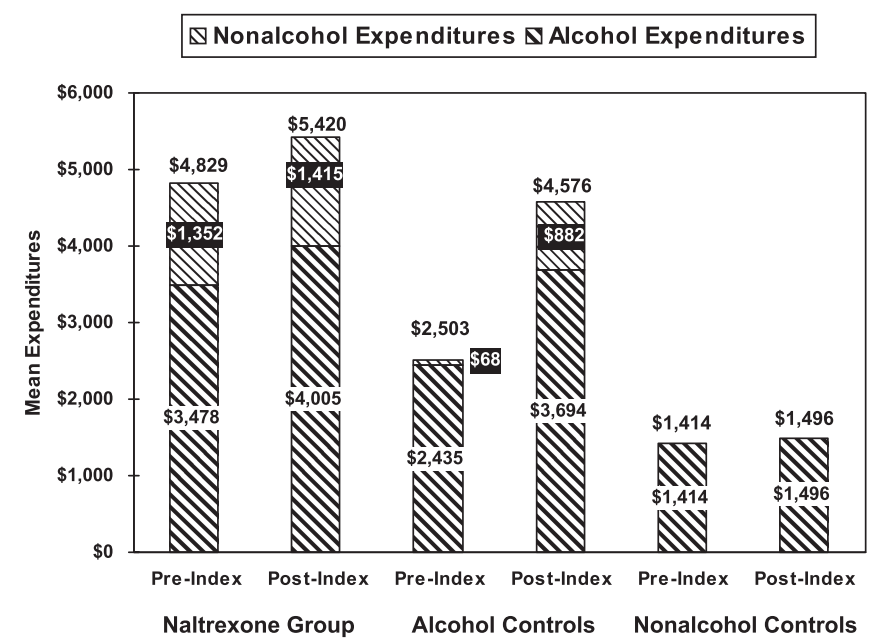

Fig. 1. Mean alcohol and nonalcohol expenditures during the 6-month pre-index and post-index periods.

expenditures than either the alcohol controls or the nonalcohol controls ( $\$ 4,829$ vs. $\$ 2,503, p<0.001$ and vs. $\$ 1,404$, $p<0.001$, respectively). In the postindex period, mean alcohol-related expenditures were higher in the naltrexone group than in the alcohol controls $(\$ 1,415$ vs. $\$ 882$, $p<0.001$ ). (These expenditures included $\$ 268$ for naltrexone, disulfiram, or acamprosate prescriptions; data not shown.) Nonalcohol-related expenditures did not differ significantly between these two groups ( $\$ 4,005$ vs. $\$ 3,694$, $p=0.16$ ). Mean total expenditures for the naltrexone group were higher than for both control groups $(\$ 5,420$ vs. $\$ 4,576$ for alcohol controls and $\$ 1,496$ for nonalcohol controls, $p<0.001)$.

Comparing the difference between pre-index period and postindex period expenditures, however, indicated that the alcohol-related expenditures for the naltrexone group were only $\$ 63$ dollars higher in the postindex period than in the pre-index period, while the difference for the controls with an alcohol-related diagnosis was an increase of $\$ 814$ $(p<0.0001)$. The smaller pre-post difference for the naltrexone group is especially notable given that these patients incurred postindex expenditures for naltrexone prescriptions, while the alcohol controls had no such expenditures in the postindex period. The trends for nonalcohol-related and total expenditures were similar to those for alcohol-related expenditures.

Specific types of service utilization are shown in Table 2. As can be seen there, a small proportion $(<5 \%)$ of patients

Table 2. Healthcare Utilization of Naltrexone Patients and Controls

\begin{tabular}{|c|c|c|c|c|c|}
\hline & $\begin{array}{l}\text { Naltrexone group } \\
\quad(N=1,138)\end{array}$ & $\begin{array}{l}\text { Alcohol controls } \\
(N=3,411)\end{array}$ & $p$-value ${ }^{a}$ & $\begin{array}{c}\text { Nonalcohol controls } \\
(N=3,410)\end{array}$ & $p$-value \\
\hline \multicolumn{6}{|c|}{ Pre-index period $N(\%)$ with any utilization } \\
\hline \multicolumn{6}{|c|}{ Alcohol-related } \\
\hline Inpatient admission & $241(21.2)$ & $29(0.9)$ & $<0.0001$ & - & - \\
\hline Inpatient detoxification & $142(12.5)$ & $17(0.5)$ & $<0.0001$ & - & - \\
\hline ED visit & $202(17.8)$ & $21(0.6)$ & $<0.0001$ & - & - \\
\hline Outpatient office visit & $570(50.1)$ & $179(5.3)$ & $<0.0001$ & - & - \\
\hline Outpatient detoxification & $11(1.0)$ & $1(0.03)$ & 0.0643 & - & - \\
\hline Outpatient psychotherapy & $189(16.6)$ & $103(3.0)$ & $<0.0,001$ & - & - \\
\hline Oral naltrexone & $52(4.6)$ & - & - & - & - \\
\hline Disulfiram/acamprosate ${ }^{c}$ & $25(2.2)$ & - & - & - & - \\
\hline \multicolumn{6}{|l|}{ Nonalcohol-related } \\
\hline Inpatient admission & $300(26.4)$ & $395(11.6)$ & $<0.0001$ & $92(2.7)$ & $<0.0001$ \\
\hline ED visit & $420(36.9)$ & $821(24.1)$ & 0.0012 & $265(7.8)$ & $<0.0001$ \\
\hline Outpatient office visit & $1,045(91.8)$ & $2,724(79.9)$ & 0.0719 & $2,311(67.8)$ & 0.0002 \\
\hline Outpatient prescriptions & $1,039(91.3)$ & $2,593(76.0)$ & 0.0199 & $2,181(64.0)$ & $<0.0001$ \\
\hline \multicolumn{6}{|c|}{ Post-index period $N(\%)$ with any utilization } \\
\hline \multicolumn{6}{|l|}{ Alcohol-related } \\
\hline Inpatient admission & $100(8.8)$ & $338(9.9)$ & 0.6092 & - & - \\
\hline Inpatient detoxification & $61(5.4)$ & $188(5.5)$ & 0.9280 & _ & - \\
\hline ED visit & $98(8.6)$ & $497(14.6)$ & 0.0148 & - & - \\
\hline Outpatient office visit & $596(52.4)$ & $2,062(60.5)$ & 0.1340 & - & - \\
\hline Outpatient detoxification & $3(0.3)$ & $15(0.4)$ & 0.6790 & - & - \\
\hline Outpatient psychotherapy & $274(24.1)$ & $734(21.5)$ & 0.4553 & - & - \\
\hline Oral naltrexone & $1,138(100)$ & - & - & - & - \\
\hline Disulfiram or acamprosate & $135(11.9)$ & _ & - & _ & - \\
\hline \multicolumn{6}{|l|}{ Nonalcohol-related } \\
\hline Inpatient admission & $182(16.0)$ & $771(22.6)$ & 0.0360 & $75(2.2)$ & $<0.0001$ \\
\hline ED visit & $319(28.0)$ & 1,257 (36.9) & 0.0310 & $273(8.0)$ & $<0.0001$ \\
\hline Outpatient office visit & $1,058(93.0)$ & $3,025(88.7)$ & 0.5308 & $2,345(68.8)$ & 0.0002 \\
\hline Outpatient prescriptions & $1,091(95.9)$ & $2,820(82.7)$ & 0.0516 & $2,205(64.7)$ & $<0.0001$ \\
\hline
\end{tabular}

${ }^{a}$ Comparison of naltrexone group and alcohol controls.

${ }^{b}$ Comparison of naltrexone group and nonalcohol controls.

${ }^{C}$ Patients in the naltrexone group had no naltrexone, disulfiram, or acamprosate claims in the 3 months pre-index, but may have used these medications prior to that time. 
in the naltrexone group had claims for oral naltrexone or disulfiram/acamprosate in the pre-index period, but more than 3 months prior to the index date. Given the requirement that controls be free of these medications, no such use was seen among the alcohol controls. One-half of the naltrexone group had an alcohol-related physician office visit in the preperiod, compared to only $5 \%$ of the alcohol controls $(p<0.0001)$. The corresponding rates for outpatient alcohol-related psychotherapy visits were 17 and $3 \%(p<$ 0.0001). A larger proportion of the naltrexone group also utilized nonalcohol-related services in the pre-index period than either of the control groups, with nearly all comparisons being highly statistically significant.

In the postindex period, however, only the rate of alcohol-related ED visits differed significantly between these groups, with the alcohol controls being more likely to experience an alcohol-related ED visit $[15 \%$ compared to only $9 \%$ of naltrexone patients $(p=0.015)]$. In the postindex period, alcohol controls also were more likely than naltrexone patients to have nonalcohol-related inpatient admissions and $\mathrm{ED}$ visits $(p=0.036$ and $p=0.031$, respectively). The percentage of patients with outpatient physician visits or prescription drug use was higher in the naltrexone group than the alcohol controls, although not significantly so $(p=0.53$ and $p=0.052$, respectively). In the postindex period, nonalcohol controls used significantly fewer nonalcohol-related services than did naltrexone patients (all categories of services showing highly significant differences).

\section{Multivariate Analyses}

After adjusting for baseline differences, we observed that the trend over time in expenditures was significantly higher for alcohol controls than for the naltrexone group. The average medical care expenditures for the naltrexone group before and after treatment relative to the alcohol controls with the same characteristics were predicted from the model using simulations. The expenditure trend estimates are noted in Table 3. They suggest that average alcohol-related, nonalcohol-related, and total expenditures for the naltrexone group increased over time by about $\$ 58, \$ 654$, and $\$ 732$, respectively, per patient, while average expenditures for alcohol controls increased by about $\$ 815, \$ 1,590$, and $\$ 2,559$, respectively. Thus, the use of naltrexone resulted in an overall

Table 3. Change in Expenditures Pre- and Postindex After Controlling for Confounders in the Multivariate Analysis

ECM difference in difference model: gamma with log link

\begin{tabular}{lcc}
\hline Expenditure type & $\begin{array}{c}\text { Naltrexone group } \\
\text { vs. alcohol controls }\end{array}$ & $\begin{array}{c}\text { Naltrexone group vs. } \\
\text { nonalcohol controls }\end{array}$ \\
\hline $\begin{array}{l}\text { Alcohol-related } \\
\text { Nonalcohol-related }\end{array}$ & $-\$ 758$ & $\$ 58$ \\
Total & $-\$ 937$ & $\$ 503$ \\
\hline
\end{tabular}

savings in total healthcare expenditures of about $\$ 1,827$ per patient (i.e., $\$ 732-\$ 2,559=-\$ 1,827$; $p$-value $<0.0001$ ). Results using alternate modeling strategies, such as ordinary least squares regression, were similar, although the magnitude of the savings estimate in total healthcare expenditures associated with naltrexone use was somewhat lower, ranging from $\$ 1,442$ to $\$ 1,483$.

\section{DISCUSSION}

Univariate comparisons indicated that the three groups were matched successfully on demographic measures. Although overall the study groups had a low level of comorbidity, the descriptive results identified some differences between the naltrexone and control groups on these measures, which were controlled for in the multivariate analysis.

Naltrexone patients had higher mean total healthcare expenditures than patients without an alcohol-related disorder, in part because of the expenditures for naltrexone and alcohol-related services. A similar comparison showed significantly higher mean nonalcohol-related expenditures, consistent with prior findings that the healthcare impact of alcohol-related disorders extends beyond alcohol-related services (Parthasarathy et al., 2001).

Naltrexone use was associated with a reduction in both alcohol-related and nonalcohol-related healthcare expenditures among patients with alcohol-related diagnoses. The savings occurred over a relatively short timeframe -6 months after the initiation of naltrexone therapy - and were significant despite the fact that naltrexone patients incurred costs for naltrexone pharmacotherapy that the controls did not incur.

Following an initial increase in costs, which is thought to reflect a pent-up need for health care that is finally being met in the course of early recovery (Holder and Blose, 1992), there was a decrease in the use of more costly, intensive treatment services, such as ED visits and inpatient stays, which translated overall into a smaller increase in costs than was seen among the alcohol controls. The detection of a differential economic benefit with naltrexone within only 6 months of its initiation may reflect a benefit of adding the medication to alcoholism treatment not only in terms of cost but also in time.

Figure 1 shows that the greater increase in healthcare costs over time in the alcohol controls were because of the lower alcohol-related and nonalcohol-related costs in this group than in the naltrexone group during the pre-period. During the postperiod, alcohol-related and nonalcohol-related costs were comparable in the two groups. We would speculate that this reflects a stabilization of the naltrexone group's course. In contrast, in the absence of naltrexone, the alcohol controls may have shown the cumulative adverse medical effects of continued heavy drinking, which were reflected in both alcohol-related and alcohol-nonrelated costs. Correlation of the change in healthcare costs with data on drinking behavior, which were not available for use in this study, would make it 
possible to evaluate this hypothesis and should be the focus of subsequent studies.

The differences in healthcare utilization between the naltrexone group and the alcohol controls are also reflected in the kinds of alcohol-related services that were utilized and how these changed over time. In the preperiod, the naltrexone group made significantly more alcohol-related outpatient medical and psychotherapy visits and ED visits, consistent with the adverse consequences of heavy drinking. Interventions, which included the prescribing of naltrexone, appeared to stabilize these individuals' course. In contrast, utilization of these services by the alcohol control group, which was low during the preperiod, increased dramatically during the postperiod, consistent with cumulative adverse consequences of heavy drinking and efforts by their healthcare practitioners to treat their alcohol-related disorders.

In short, the naltrexone group and the alcohol controls, despite being matched on a variety of demographic and clinical variables during the preperiod, differed on a variety of measures of healthcare utilization. It appears that the naltrexone group was prescribed this opioid antagonist medication in the context of a variety of alcohol-related healthcare services. Using utilization data, it is not possible to partial out the impact of the naltrexone per se and an alternate explanation for the observed trajectories is that factors other than naltrexone (i.e., both treatment-related and possibly reflecting regression to the mean) were responsible for the differential change in healthcare utilization between the naltrexone group and the alcohol controls.

This study has a number of strengths, including the use of two comparison groups, one of which was diagnosed with an alcohol-related disorder and the other without such a diagnosis, which made it possible to evaluate temporal trends that might otherwise confound the interpretation of the findings (Holder and Blose, 1992). The use of GEE accounted for the autocorrelation that occurs among individuals with repeated measurements (Liang and Zeger, 1986). Finally, the detailed healthcare utilization information available made it possible to examine different modalities of treatment received, both alcohol-related and nonalcohol-related, resulting in a more detailed and revealing analysis of the impact of naltrexone treatment on healthcare utilization.

These results should be interpreted in light of the usual limitations of analyses using administrative data. Because this study used only information available on insurance claims, it was necessary to include patients with a variety of alcoholrelated disorders, which served as a proxy measure of an alcohol use disorder and it was not possible to stratify patients by the level of severity of their alcohol-related disorder. Because data for individuals with an oral naltrexone claim who did not meet all study inclusion and exclusion criteria were not extracted from the database, it was not possible to compare the characteristics of excluded oral naltrexone patients to the study sample. Psychiatric comorbidity may have differed by cohort, but was not assessed. Nor was it possible to ascertain patients' motivation to seek alcohol-related services. There was no way to verify that patients with an alcohol-related diagnosis did, in fact, have such a diagnosis and some patients in the control group without an alcohol-related diagnosis could have had an alcohol-related disorder that was not coded in their insurance claims. Naltrexone is also approved to treat opioid dependence, so it is possible that some individuals were prescribed naltrexone to treat that disorder, rather than an alcohol-related disorder. Because naltrexone is prescribed for opioid dependence infrequently, it is unlikely that this is an important consideration in the interpretation of the data presented here. Some patients in the naltrexone group had evidence of pre-index use of naltrexone, disulfiram, or acamprosate. Although the small number of such patients suggests that their impact on study results was minimal, this was not specifically assessed. In addition, because only services billed to insurers could be quantified, we were unable to assess modalities such as participation in Alcoholics Anonymous, so it is not known whether their utilization differed between the naltrexone and control groups.

Despite these limitations, the findings of this analysis are notable. They suggest that there is a cost savings to payers of making treatment with naltrexone available to their enrollees. The pre-to-post decrease in nonalcohol-related healthcare utilization seen among the naltrexone patients but not among the alcohol controls indicates that naltrexone treatment may also decrease the overall burden on the healthcare system.

It may be feasible to derive more benefit from naltrexone treatment than is evident from these results. Rates of adherence to treatment with oral naltrexone have been shown to be poor, with most patients unable to complete a clinical course of treatment (Harris et al., 2004; Hermos et al., 2004; Kranzler et al., 2008). A prior analysis of data from the naltrexone group included in this study showed that of patients prescribed oral naltrexone, nearly $86 \%$ did not fill their prescriptions for at least $80 \%$ of the days over a 6 -month period and approximately $50 \%$ failed to refill even one prescription (Kranzler et al., 2008). Patients who persisted in refilling at least $80 \%$ of the prescriptions during the 6 -month period showed significantly lower rates of use of intensive medical services compared to patients who did not meet this persistence criterion, including fewer ED contacts, hospital admissions, and inpatient alcohol detoxifications. This finding has relevance for the question of whether the different trajectories of the naltrexone group and the alcohol controls were because of treatment with the medication. Although it is possible that naltrexone was prescribed to individuals who were perceived to have a better prognosis, the low persistence rate for naltrexone argues against this interpretation. Further, the higher alcohol-related healthcare costs in the naltrexone group than the alcohol controls during the pre-index period is inconsistent with the notion that the naltrexone group was more stable during this period than the alcohol controls.

These observations underscore the potential importance of initiating medication treatment for alcohol dependence and of optimizing medication adherence. Such efforts can include behavioral approaches (e.g., daily observed pill 
administration) that have been shown to enhance adherence (Cramer et al., 2003; O'Farrell et al., 1995; Pettinati et al., 2000; Starosta et al., 2006) or long-acting preparations such as extended-release injectable naltrexone (Garbutt et al., 2005).

Finally, because of the relatively short duration of monitoring, the observed effects on healthcare utilization may be an underestimate of the actual impact. Additional studies using a longer duration of monitoring appear warranted to estimate more accurately the cost offset resulting from the use of naltrexone to treat alcoholism. Particular attention is also required to differentiate the effects of the medication from co-occurring interventions that follow from the recognition by healthcare practitioners that an individual's drinking is problematic.

\section{ACKNOWLEDGMENTS}

Supported by Alkermes, Inc., Cephalon, Inc., and grant K24 AA13736 (to HRK) from the National Institute on Alcohol Abuse and Alcoholism.

\section{REFERENCES}

Caetano R, Cunradi C (1997) Alcohol dependence: a public health perspective. Addiction 97:633-645.

Cohen E, Feinn R, Arias A, Kranzler HR (2007) Alcohol treatment utilization: findings from the National Epidemiologic Survey on Alcohol and Related Conditions. Drug Alcohol Depend 86:214-221.

Cramer J, Rosenheck R, Kirk G, Krol W, Krystal J (2003) VA Naltrexone Study Group 42: Medication compliance feedback and monitoring in a clinical trial: predictors and outcomes. Value Health 6:566-573.

D'Hoore W, Bouckaert A, Tilquin C (1996) Practical considerations on the use of the Charlson comorbidity index with administrative data bases. J Clin Epidemiol 49:1429-1433.

Garbutt JC, Kranzler HR, O'Malley SS, Gastfriend DR, Pettinati HM, Silverman BL, Loewy JW, Ehrich EW (2005) Efficacy and tolerability of long-acting injectable naltrexone for alcohol dependence: a randomized controlled trial. JAMA 293:1617-1625.

Grant BF, Dawson DA, Stinson FS, Chou SP, Dufour MC, Pickering RP (2004) The 12-month prevalence and trends in DSM-IV alcohol abuse and dependence: United States, 1991-1992 and 2001-2002. Drug Alcohol Depend 74:223-234.

Harris KM, DeVries A, Dimidjian K (2004) Datapoints: Trends in naltrexone use among members of a large private health plan. Psychiatr Serv 55:221.

Harwood H (2000) Updating Estimates of the Economic Costs of Alcohol Abuse in the United States: Estimates, Update Methods, and Data. Report prepared by The Lewin Group for the National Institute on Alcohol Abuse and Alcoholism. Based on estimates, analyses, and data reported in Harwood, H, Fountain D, Livermore G. The Economic Costs of Alcohol and Drug Abuse in the United States 1992. Report prepared for the National Institute on Drug Abuse and the National Institute on Alcohol Abuse and Alcoholism, National Institutes of Health, Department of Health and Human Services. NIH Publication No. 98-4327. Rockville, MD: National Institutes of Health, 1998. Available from: http://pubs. niaaa.nih.gov/publications/economic-2000/alcoholcost.PDF.
Harwood H, Fountain D, Livermore G (1998) The economic costs of alcohol and drug abuse in the United States 1992. Report prepared for the National Institute on Drug Abuse and the national Institute on Alcohol Abuse and Alcoholism, Nation Institutes of Health, Department of Health and Human Services. National Institute of Health Publication No. 98-4327. Rockville, MD: National Institutes of Health. Available from: http://www.nida.nih. gov/Economics-Costs/Index.html.

Hermos JA, Young MM, Gagnon DR, Fiore LD (2004) Patterns of dispensed disulfiram and naltrexone for alcoholism treatment in a veteran patient population. Alcohol Clin Exp Res 28:1229-1235.

Holder HD (1998) Cost benefits of substance abuse treatment: an overview of results from alcohol and drug abuse. J Ment Health Policy Econ 1:23-29.

Holder HD, Blose JO (1992) The reduction of health care costs associated with alcoholism treatment: a 14-year longitudinal study. J Stud Alcohol 53:293302.

Kranzler H, Stephenson J, Montejano L, Wang S, Gastfriend D (2008) Persistence with oral naltrexone for alcohol treatment: implications for healthcare utilization. Addiction 103:1801-1808.

Liang K, Zeger S (1986) Longitudinal data analysis using generalized linear models. Biometrika 73:13-22.

Mandrekar JN, Mandrekar SJ (2004). An introduction to matching and its application using SAS ${ }^{\circledR}$, In: SAS Institute Inc. Proceedings of the TwentyNinth Annual SAS ${ }^{\circledR}$ Users Group International Conference. SAS Institute Inc., Cary, NC.

Mojtabai R, Olfson M, Mechanic D (2002) Perceived need and help-seeking in adults with mood, anxiety, or substance use disorders. Arch Gen Psychiatry 59:77-84.

O'Farrell TJ, Allen JP, Litten RZ (1995) Disulfiram (antabuse) contracts in treatment of alcoholism. NIDA Res Monogr 150:65-91.

Parthasarathy S, Weisner C, Hu TW, Moore C (2001) Association of outpatient alcohol and drug treatment with health care utilization and cost: revisiting the offset hypothesis. J Stud Alcohol 62:89-97.

Pettinati HM, Meyers K, Evans BD, Ruetsch CR, Kaplan FN, Jensen JM, Hadley TR (1999) Inpatient alcohol treatment in a private healthcare setting: which patients benefit and at what cost? Am J Addict 8:220-233.

Pettinati HM, Volpicelli JR, Pierce JD Jr, O'Brien CP (2000) Improving naltrexone response: an intervention for medical practitioners to enhance medication compliance in alcohol dependent patients. J Addict Dis 19:7183.

Putnam KG, Buist DS, Fishman P, Andrade SE, Boles M, Chase GA, Goodman MJ, Gurwitz JH, Platt R, Raebel MA, Arnold Chan K (2002) Chronic disease score as a predictor of hospitalization. Epidemiology 13:340-346.

Rabinowitz J, Gross R, Feldman D (1999) Correlates of a perceived need for mental health assistance and differences between those who do and do not seek help. Soc Psychiatry Psychiatr Epidemiol 34:141-146.

Regier DA, Narrow WE, Rae DS, Manderscheid RW, Locke BZ, Goodwin FK (1993) The de facto US mental and addictive disorders service system. Epidemiologic catchment area prospective 1-year prevalence rates of disorders and services. Arch Gen Psychiatry 50:85-94.

Starosta AN, Leeman RF, Volpicelli JR (2006) The BRENDA model: integrating psychosocial treatment and pharmacotherapy for the treatment of alcohol use disorders. J Psychiatr Pract 12:80-89.

Valderas JM, Starfield MDB, Sibbald B, Salisbury C, Roland M (2009) Defining comorbidity: Implications for understanding health and health services. Ann Fam Med 7:357-363.

Von Korff M, Wagner E, Saunders K (1992) A chronic disease score from automated pharmacy data. J Clin Epidemiol 45:197-203.

Wu L, Ringwalt CL, Williams CE (2003) Use of substance abuse treatment services by persons with mental health and substance use problems. Psychiatr Serv 54:363-369. 